\title{
PRESCRIBING RICCI CURVATURE ON COMPLEXIFIED SYMMETRIC SPACES
}

\author{
Roger Bielawski
}

The aim of this note is to prove the existence of invariant Ricci-flat Kähler metrics on complexifications of symmetric spaces of compact type. Before stating the result, let us fix the notation.

Let $(M, g)$ be a Riemannian symmetric space of compact type and $p$ a point in $M$. Let $G$ be the identity component of the isometry group of $(M, g)$ and let $K$ be the stabiliser of $p$ in $G$. Then $M \simeq G / K$ and the complexification of $M$ is $T M$ with the adapted complex structure [7] that can be identified with $G^{\mathbb{C}} / K^{\mathbb{C} 1}$. We are going to prove

Theorem 1. Let $(M, g)$ be an irreducible symmetric space of compact type. Let $G$ and $K$ be as above and suppose that $K$ is connected. Let $\rho$ be a real exact $G$ invariant $(1,1)$-form on the complexification $T M \simeq G^{\mathbb{C}} / K^{\mathbb{C}}$. Then there exists a G-invariant Kähler metric on TM whose Ricci form is $\rho$.

Remark. The Kähler form obtained in Theorem 1 is exact.

The above result has been proved in [9] for symmetric spaces of rank 1 and in [2] for compact groups, i.e. for the case when $G=K \times K$ and $K$ acts diagonally. For hermitian symmetric spaces and $\rho=0$, Theorem 1 has also been known [4].

The proof given here is quite different from that given for group manifolds in [2]. We show that the complex Monge-Ampère equation on $G^{\mathbb{C}} / K^{\mathbb{C}}$ reduces, for $G$-invariant functions, to a real Monge-Ampère equation on the dual symmetric space $G^{*} / K$. We also show that the Monge-Ampère operator on non-compact symmetric spaces has a radial part, i.e. it is equal, for $K$-invariant functions, to another Monge-Ampère operator on the maximal abelian subspace of $G^{*} / K$. These facts, together with the theorem on $K$-invariant real Monge-Ampère equations proved in [3], yield Theorem 1.

\section{Riemannian symmetric spaces of non-compact type}

Here we recall some facts about the geometry of Riemannian symmetric spaces. The standard reference for this section is [6].

Let $M=G / K$ be a symmetric space of compact type with $K$ connected, and let $G^{*} / K$ be its dual. If $\mathfrak{g}, \mathfrak{g}^{*}$ and $\mathfrak{k}$ denote the Lie algebras of $G, G^{*}$ and

\footnotetext{
Received February 25, 2004.

${ }^{1}$ The complexification of a compact connected Lie group $G$ is the connected group $G^{\mathrm{C}}$ whose Lie algebra is the complexification of the Lie algebra of $G$ and which satisfies $\pi_{1}\left(G^{\mathbb{C}}\right)=\pi_{1}(G)$.
} 
$K$, then $\mathfrak{g}=\mathfrak{k} \oplus \mathfrak{p}, \mathfrak{g}^{*}=\mathfrak{k} \oplus i \mathfrak{p}$, where $[\mathfrak{k}, \mathfrak{p}] \subset \mathfrak{p}$ and $[\mathfrak{p}, \mathfrak{p}] \subset \mathfrak{k}$. The restriction of the Killing form to $i \mathfrak{p}$ is positive definite and induces the Riemannian metric of $G^{*} / K$. Moreover, the Riemannian exponential mapping provides a diffeomorphism between $\mathfrak{p}$ and $G^{*} / K$. This can be viewed as the map:

$$
p \mapsto e^{i p} K
$$

where $p \in \mathfrak{p}$ and $e$ is the group-theoretic exponential map for $G^{*}$. Thus we have two $K$-invariant Riemannian metrics on $\mathfrak{p} \simeq \mathbb{R}^{n}$ : the Euclidean one given by the Killing form, and the negatively curved one given by the diffeomorphism (1.1).

Let $\mathfrak{a}$ be a maximal abelian subspace of $\mathfrak{p}$ and $\mathfrak{l}$ its centraliser in $\mathfrak{k}$. Let $\Sigma$ be the set of restricted roots and $\Sigma^{+}$the set of restricted positive roots. For each $\alpha \in \Sigma$, let $\mathfrak{p}_{\alpha}\left(\right.$ resp. $\left.\mathfrak{k}_{\alpha}\right)$ denote the subspace of $\mathfrak{p}$ (resp. of $\mathfrak{k}$ ) where each $(\operatorname{ad} H)^{2}, H \in \mathfrak{a}$, acts with eigenvalue $\alpha(H)^{2}$. We have the direct decompositions

$$
\mathfrak{p}=\mathfrak{a}+\sum_{\alpha \in \Sigma^{+}} \mathfrak{p}_{\alpha}, \quad \mathfrak{k}=\mathfrak{l}+\sum_{\alpha \in \Sigma^{+}} \mathfrak{k}_{\alpha} .
$$

Let $\mathfrak{a}^{+}$be an open Weyl chamber and let $\mathfrak{p}^{\prime}$ be the union of $K$-orbits of points in $\mathfrak{a}^{+}$. Any $K$ orbit in $\mathfrak{p}^{\prime}$ is isomorphic to $K / L$ where the Lie algebra of $L$ is $\mathfrak{l}$. Moreover, we have the diffeomorphism:

$$
\mathfrak{a}^{+} \times K / L \rightarrow \mathfrak{p}^{\prime}, \quad(h, k) \mapsto \operatorname{Ad}(k) h .
$$

We now wish to write the two $K$-invariant metrics on $\mathfrak{p}$ in coordinates given by this diffeomorphism. Let $\sum d r_{i}^{2}$ be the Killing metric on $\mathfrak{a}^{+}$(the $r_{i}$ can be viewed as $K$-invariant functions on $\left.\mathfrak{p}^{\prime}\right)$. For each $\mathfrak{k}_{\alpha}$, choose a basis $X_{\alpha, m}(m$ runs from 1 to twice the multiplicity of $\alpha$ ) of vectors orthonormal for the Killing form and denote by $\theta_{\alpha, m}$ the corresponding basis of invariant 1-forms on $K / L$. We have

Proposition 1.1. Let $g_{0}$ be the Euclidean metric on $\mathfrak{p}$, given by the restriction of the Killing form, and let $g$ be the negatively curved symmetric metric on $\mathfrak{p}$ given by the diffeomorphism (1.1). Then, under the diffeomorphism (1.3) the metrics $g_{0}$ and $g$ can be written in the form

$$
\sum_{i} d r_{i}^{2}+\sum_{(\alpha, m)} F(\alpha(r)) \theta_{(\alpha, m)}^{2}
$$

where $F(z)=z^{2}$ for $g_{0}$, and $F(z)=\sinh ^{2}(z)$ for $g$.

Proof. Since all these metrics are $K$-invariant, it is enough to compute them at points of $\mathfrak{a}^{+}$. Let $H$ be such a point and let $(h, \rho), h \in \mathfrak{a}, \rho \in T_{[1]} K / L$, be a tangent vector to $\mathfrak{a}^{+} \times K / L$ at $(H,[1])$. The vector $\rho$ can be identified with an element of $\sum \mathfrak{k}_{\alpha} \subset \mathfrak{k}$. The corresponding (under (1.3)) tangent vector at $H \in \mathfrak{p}^{\prime}$ is $h+[\rho, H]$. Computing the Killing form of this vector yields the formula (1.4) with $F(z)=z^{2}$ for $g_{0}$. The formula for $g$ follows from a similar computation, using the expression for the differential of the map (1.1) given in [6], Theorem IV.4.1. 


\section{Monge-Ampère equation on symmetric spaces}

Let $(M, g)$ be a Riemannian manifold and $u: M \rightarrow \mathbb{R}$ a smooth function. Then the Hessian of $u$ is the symmetric $(0,2)$-tensor $D d u$ where $D$ is the LeviCivita connection of $g$. In local coordinates $x_{i}, D d u$ is represented by the matrix

$$
H_{i j}=\frac{\partial^{2} u}{\partial x_{i} \partial x_{j}}-\sum_{k} \Gamma_{i j}^{k} \frac{\partial u}{\partial x_{k}} .
$$

We say that the function $u$ is $g$-convex (resp. strictly $g$-convex), if $D d u$ is nonnegative (resp. positive) definite. The Monge-Ampère equation on the manifold $(M, g)$ is then

$$
\mathbf{M}_{g}(u):=(\operatorname{det} g)^{-1} \operatorname{det} D d u=f
$$

where $f$ is a given function.

Let $\left(G^{*} / K, g\right)$ be a symmetric space of non-compact type given by a Cartan decomposition $\mathfrak{g}^{*}=\mathfrak{k}+i \mathfrak{p}$. As in the previous section, we identify $M=G^{*} / K$ with $\mathfrak{p}$ and denote by $g_{0}$ the (flat) metric given by restricting the Killing form to $\mathfrak{p}$. We have:

Theorem 2.1. Let $M \simeq \mathfrak{p}$ be a symmetric space of noncompact type and let $u$ be a $K$-invariant (smooth) function on $M$. Then

(1) $u$ is $g$-convex if and only if $u$ is $g_{0}$-convex (i.e. convex in the usual sense on $\mathfrak{p})$.

(2) The following equality of Monge-Ampère operators holds:

$$
\mathbf{M}_{g}(u)=F \cdot \mathbf{M}_{g_{0}}(u),
$$

where $F: M \rightarrow \mathbb{R}$ is a positive $K$-invariant smooth function depending only on $M$.

We have proved in [3] a theorem on the existence and regularity of $K$-invariant solutions to Monge-Ampère equations on $\mathbb{R}^{n}$. From this we immediately obtain

Corollary 2.2. Let $\left(G^{*} / K, g\right)$ be an irreducible symmetric space of noncompact type and let $f$ be a positive smooth $K$-invariant function on $G^{*} / K$. Then the Monge-Ampère equation (2.2) has a global smooth $K$-invariant strictly $g$-convex solution.

We shall now prove Theorem 2.1. In fact we shall prove it in the following, more general situation. Suppose that we are given a $K$-invariant metric on $\mathfrak{p}$ whose pullback under (1.3) can be written as (cf. (1.4)):

$$
\sum_{i} d r_{i}^{2}+\sum_{(\alpha, m)} F_{(\alpha, m)}(\alpha(r)) \theta_{(\alpha, m)}^{2},
$$

where $F_{(\alpha, m)}: \mathbb{R} \rightarrow \mathbb{R}$ are smooth functions vanishing at the origin such that $z^{-1} \frac{d F(\alpha, m)}{d z}$ is smooth and positive everywhere. Proposition 1.1 implies that the symmetric metric on $G^{*} / K$ is of this form. We claim that Theorem 2.1 holds for any metric $g$ of the form (2.3). 
In order to simplify the notation, let us write $j$ for the index $(\alpha, m)$ and $\alpha_{j}$ for $\alpha$ if $j=(\alpha, m)$. The metric $g$ can be now written as

$$
\sum_{i} d r_{i}^{2}+\sum_{j} F_{j}\left(\alpha_{j}(r)\right) \theta_{j}^{2}
$$

We recall the following formula:

$$
2 D d u=L_{\nabla u} g
$$

where $L$ is the Lie derivative and $\nabla u$ is the gradient of $u$ with respect to the metric $g$. On the other hand, for any $(0,2)$-tensor $g$ and vector fields $X, Y, Z$, we have:

$$
\left(L_{X} g\right)(Y, Z)=X . g(Y, Z)-g([X, Y], Z)-g(Y,[X, Z]) .
$$

We now compute $L_{\nabla u} g$ on $\mathfrak{p}^{\prime}$ with respect to the basis vector fields $\partial / \partial r_{i}, X_{j}$, where $X_{j}$ are dual to $\theta_{j}$. Here $u$ is a $K$-invariant function. The gradient of $u$ is just $\sum \frac{\partial u}{\partial r_{i}} \frac{\partial}{\partial r_{i}}$, in particular it is independent of the functions $F_{j}$. It follows immediately that $\left(L_{\nabla u} g\right)\left(\partial / \partial r_{i}, X_{j}\right)=0$ and that the matrix $\left(L_{\nabla u} g\right)\left(X_{j}, X_{k}\right)$ is equal to $\nabla u . g\left(X_{j}, X_{k}\right)$ and hence it is diagonal with the $(j j)$-entry equal to

$$
\nabla u\left(F_{j}\left(\alpha_{j}(r)\right)\right)=\left.\frac{d F_{j}}{d z}\right|_{z=\alpha_{j}(r)} \alpha_{j}\left(\nabla_{0} \bar{u}\right) .
$$

Here $\nabla_{0} \bar{u}=\sum \frac{\partial u}{\partial r_{i}} \frac{\partial}{\partial r_{i}}$ is the gradient of $u$ restricted to the Euclidean space $\mathfrak{a}=\mathbb{R}^{n}$ in coordinates $r_{i}$, and viewed as a map from $\mathbb{R}^{n}$ to itself.

Theorem 2.1 with the more general metric (2.3) follows easily with the function $F$ given explicitly by

$$
F=\frac{\prod \alpha_{j}(r)}{\prod F_{j}\left(\alpha_{j}(r)\right)} \prod\left(\frac{1}{2} \frac{d F_{j}}{d z}\right)_{z=\alpha_{j}(r)} .
$$

Observe that the assumptions on the $F_{j}$ guarantee that $F$ extends to a smooth positive function on $\mathfrak{p}$.

\section{Proof of the Main Theorem}

Let $(M, g)$ be a Riemannian symmetric space of compact type, $G$ its isometry group, and $K \subset G$ the stabiliser group of a point. There is a canonical isomorphism between $G^{\mathbb{C}} / K^{\mathbb{C}}$ and $G \times_{K} \mathfrak{p}$ (i.e. the tangent bundle of $G / K$ ) given by the map:

$$
G \times \mathfrak{p} \rightarrow G^{\mathbb{C}} \rightarrow G^{\mathbb{C}} / K^{\mathbb{C}}, \quad(g, p) \mapsto g e^{i p} .
$$

This isomorphism can be viewed in many ways: as an example of Mostow fibration [8], as given via Kähler reduction of $G^{\mathbb{C}} \simeq G \times \mathfrak{g}$ by the group $K$ [5], or as given by the adapted complex structure construction [7] which provides a canonical diffeomorphism between the tangent bundle of $G / K$ and a complexification of $G / K$. In any case it provides a fibration

$$
\pi: G^{\mathbb{C}} / K^{\mathbb{C}} \rightarrow G / K
$$


The fibers of this projection can be identified with $\mathfrak{p}$ via the map (3.1). In particular, the fiber over [1] is given by the $K^{\mathbb{C}}$-orbits of elements $e^{i p}, p \in \mathfrak{p}$. We shall relate $G$-invariant plurisubharmonic functions on $G^{\mathbb{C}} / K^{\mathbb{C}}$ to convex functions on this fiber (see [1] for a different approach to this).

For a function $w$ on a complex manifold one defines its Levy form $L w$ to be the Hermitian $(0,2)$ tensor given in local coordinates as

$$
\frac{\partial^{2} w}{\partial z_{k} \partial \bar{z}_{l}} d z_{k} \otimes d \bar{z}_{l}
$$

This form does not depend on the choice of local coordinates. We shall compute this form for a $G$-invariant function $w$ on $G^{\mathbb{C}} / K^{\mathbb{C}}$. It is enough to compute it at points $e^{i p}, p \in \mathfrak{p}$. First of all, we choose local holomorphic coordinates at such a point:

Lemma 3.1. In a neighbourhood of a point $e^{i p}, p \in \mathfrak{p}$, complex coordinates are provided by the map $\mathfrak{p}^{\mathbb{C}} \rightarrow G^{\mathbb{C}} \rightarrow G^{\mathbb{C}} / K^{\mathbb{C}},(a+i b) \mapsto e^{a+i b} e^{i p}$.

Proof. We have to show that the map $(a+i b) \mapsto e^{a+i b} e^{i p} K^{\mathbb{C}}$ has a non-singular differential at 0 . This is equivalent to $\left(\operatorname{ad} e^{-i p}\right) u \notin \mathfrak{k}^{\mathbb{C}}$ for $u \in \mathfrak{p}^{\mathbb{C}}$. We have

$$
\left(\operatorname{ad} e^{-i p}\right) u=e^{\operatorname{ad}(-i p)} u=\cosh (\operatorname{ad}(-i p)) u+\sinh (\operatorname{ad}(-i p)) u,
$$

where the first term of the sum lies in $\mathfrak{p}^{\mathbb{C}}$ and the second one in $\mathfrak{k}^{\mathbb{C}}$. To show that the first term does not vanish recall that $(\operatorname{ad}(-i p))^{2}$ has all eigenvalues nonnegative.

We now have:

Lemma 3.2. In the complex coordinates $z=a+i b$ given by the previous lemma, the Levy form (3.3) of a G-invariant function $w$ satisfies the equation:

$$
\left(\frac{\partial^{2} w}{\partial z_{k} \partial \bar{z}_{l}}\right)_{\substack{a=0 \\ b=0}}=\frac{1}{4} \frac{\partial^{2}}{\partial b_{k} \partial b_{l}} w\left(e^{i b} e^{i p}\right)_{b=0} .
$$

Proof. The polar decomposition of $G^{\mathbb{C}}$ implies that $e^{a+i b}$ can be uniquely written as $g e^{i y}$, where $g \in G$ and $y \in \mathfrak{g}$. Any $G$-invariant function on $G^{\mathbb{C}} / K^{\mathbb{C}}$ in a neighbourhood of $e^{i p}$ is a function of $y$ only. On the other hand, as $e^{2 i y}=$ $\left(e^{x} e^{i y}\right)^{*}\left(e^{x} e^{i y}\right)=e^{-a+i b} e^{a+i b}$, it follows from the Campbell-Hausdorff formula that $y=b+[b, a] / 2+$ higher order terms. Hence the matrix of second derivatives in (3.3) at $e^{i p}$ (i.e. at $a=0, b=0$ ) is the same as the matrix of second derivatives of

$$
(a, b) \mapsto e^{\left(i b+\frac{i}{2}[b, a]\right)} e^{i p}
$$

at $a=0, b=0$. We shall now show that for a $G$-invariant function $w$ on $G^{\mathbb{C}} / K^{\mathbb{C}}$, this matrix of second derivatives is equal to the right-hand side of (3.5).

The Campbell-Hausdorff formula implies that up to order 2 in $a, b$, we have $e^{\left(i b+\frac{i}{2}[b, a]\right)}=e^{i b} e^{\left.\frac{i}{2}[b, a]\right)}$. Set $c=[b, a] / 2$, which is a point in $\mathfrak{k}$. We are going to show that modulo terms of order 2 in $c$ (hence of order 4 in $a, b$ ), $e^{i c} e^{i p}$ is equal 
to $e^{\rho} e^{i p} e^{i q}$, where $\rho \in \mathfrak{g}$ and $q \in \mathfrak{k}$ are both linearly dependent on $c$. We note that this proves the lemma, as

$$
e^{i b} e^{\rho} e^{i p} e^{i q}=e^{\rho} e^{i b+O(3)} e^{i p} e^{i q}=e^{\rho} e^{i b+O(3)} e^{i p}
$$

in $G^{\mathbb{C}} / K^{\mathbb{C}}$, where $O(3)$ denotes terms of order 3 and higher in $a, b$.

We find $q$ from the equation $\cosh \operatorname{ad}(i p)(q)=c$, which can be solved uniquely as $\cosh \operatorname{ad}(i p)$ is symmetric and positive-definite on $\mathfrak{k} \subset \mathfrak{g}$. We then put $\rho=$ $-i \sinh \operatorname{ad}(i p)(q)$. We observe that $\rho \in \mathfrak{g}$ and $e^{\rho-i c}=e^{i p} e^{-i q} e^{-i p}$, thanks to (3.4). Moreover, modulo terms quadratic in $c, e^{\rho}=e^{i c} e^{\rho-i c}$ and, consequently:

$$
e^{\rho} e^{i p} e^{i q}=e^{i c} e^{\rho-i c} e^{i p} e^{i q}=e^{i c}\left(e^{i p} e^{-i q} e^{-i p}\right) e^{i p} e^{i q}=e^{i c} e^{i p},
$$

again modulo terms quadratic in $c$. This finishes the proof of the lemma.

According to this lemma, we have to compute $\frac{\partial^{2}}{\partial b_{k} \partial b_{l}} w\left(e^{i b} e^{i p}\right)_{b=0}$. Now, since $e^{i b} e^{i p} \in G^{*}, e^{i b} e^{i p}=k e^{i z}$, where $z=z(b) \in \mathfrak{p}$ and $k \in K$. As $w$ is $G$-invariant, $w\left(e^{i b} e^{i p}\right)=w\left(e^{i z}\right)$ and therefore

$$
\frac{\partial^{2}}{\partial b_{k} \partial b_{l}} w\left(e^{i b} e^{i p}\right)_{b=0}=\frac{\partial^{2}}{\partial b_{k} \partial b_{l}} w\left(e^{i z(b)}\right)_{b=0} .
$$

Thus we compute the matrix of second derivatives of a function defined on $\exp (i \mathfrak{p})$ in the coordinates given by $b \mapsto e^{i b} e^{i p} \mapsto e^{i z(b)}$. These, however, are the geodesic coordinates at the point $e^{i p}$ in the symmetric space dual to $M$ (being translations of geodesics at [1]) and hence the matrix of second derivatives in these coordinates is equal to the Riemannian Hessian (2.1) for the symmetric metric on the dual space. If we assume that $K$ is connected, then this dual space is $G^{*} / K$, and we obtain

Theorem 3.3. Suppose that $K$ is connected. Let $w$ be a smooth $G$-invariant function on $X=G^{\mathbb{C}} / K^{\mathbb{C}}$ and let $\bar{w}$ be its restriction to the fiber $S=\exp (i \mathfrak{p})$ of (3.2) over [1]. Let $g$ denote the symmetric metric on $S \simeq G^{*} / K$. Then $w$ is (strictly) plurisubharmonic if and only if $\bar{w}$ is (strictly) g-convex. Moreover, the following equality holds:

$$
\partial \bar{\partial} \log \operatorname{det} L w=\partial \bar{\partial} \log \widehat{\mathbf{M}_{g}(\bar{w})},
$$

where $\hat{u}: X \rightarrow \mathbb{R}$ is a $G$-invariant function such that $\overline{\hat{u}}$ is a given $K$-invariant function $u$ on $S$.

We are now ready to prove Theorem 1 . Recall that $X=G^{\mathbb{C}} / K^{\mathbb{C}}$ is a Stein manifold and so if $\rho$ is an exact $(1,1)$ form on $X$, then $\rho=-i \partial \bar{\partial} h$ for some function $h$. If $\rho$ is $G$-invariant, then we can assume that $h$ is $G$-invariant. We can restrict $h$ to the fiber $S$ defined in the last theorem and thanks to Corollary 2.2 we can find a strictly $g$-convex $K$-invariant smooth solution $\bar{u}$ to the equation (2.2) with $f=e^{h}$, where the metric $g$ is the symmetric metric on $S \simeq G^{*} / K$. We can extend this solution via $G$-action to a $G$-invariant function $u$ on $X$. Theorem 3.3 implies now that $u$ is strictly plurisubharmonic and that the Ricci form of the Kähler metric with potential $u$ is $\rho$. 


\section{Acknowledgement}

This work has been supported by an Advanced Research Fellowship from the Engineering and Physical Sciences Research Council of Great Britain. I thank Hassan Azad and the anonymous referee for helpful comments which resulted in an improved presentation.

\section{References}

[1] H. Azad and J.-J. Loeb, Plurisubharmonic functions and Kählerian metrics on complexification of symmetric spaces, Indag. Math. (N.S.) 3 (1992), 365-375.

[2] R. Bielawski, Kähler metrics on $G^{\mathrm{C}}$, J. Reine Angew. Math. 559 (2003), 123-136.

[3] Entire invariant solutions to Monge-Ampère equations, Proc. Amer. Math. Soc. 132 (2004), 2679-2682.

[4] O. Biquard and P. Gauduchon, Géométrie hyperkählérienne des espaces hermitiens symétriques complexifiés, Sémin. Théor. Spectr. Géom. 16, Univ. Grenoble I, 127-173.

[5] P. Heinzner and A. Huckleberry, Analytic Hilbert quotients, in: Several complex variables (Berkeley, CA, 1995-1996), 309-349, Math. Sci. Res. Inst. Publ., 37, Cambridge University Press, Cambridge, 1999.

[6] S. Helgason, Differential geometry, Lie groups, and Symmetric spaces, Academic Press, New York, 1978.

[7] L. Lempert and R. Szőke, Global solutions of the homogeneous complex Monge-Ampère equation and complex structures on the tangent bundle of Riemannian manifolds, Math. Ann. 290 (1991), 689-712.

[8] G.D. Mostow, On covariant fiberings of Klein spaces Amer. J. Math. 77 (1955), 247-278.

[9] M.B. Stenzel, Ricci-flat metrics on the complexification of a compact rank one symmetric space, Manuscripta Math. 80 (1993), 151-163.

Department of Mathematics, University of Glasgow, Glasgow G12 8QW, UK

E-mail address: R.Bielawski@@maths.gla.ac.uk 